\title{
Erratum: Leptogenesis in the Neutrino Option
}

\author{
I. Brivio, ${ }^{a}$ K. Moffat, ${ }^{b}$ S. Pascoli, ${ }^{b}$ S.T. Petcov ${ }^{c, d}$ and J. Turner ${ }^{e}$ \\ ${ }^{a}$ Institut für Theoretische Physik, Universität Heidelberg, \\ Philosophenweg 16, 69120 Heidelberg, Germany \\ ${ }^{b}$ Institute for Particle Physics Phenomenology, Department of Physics, Durham University, \\ South Road, Durham DH1 3LE, United Kingdom \\ ${ }^{c} S I S S A / I N F N$, \\ Via Bonomea 265, I-34136 Trieste, Italy \\ ${ }^{d}$ Kavli IPMU (WPI), University of Tokyo, \\ 5-1-5 Kashiwanoha, 277-8583 Kashiwa, Japan \\ e Theoretical Physics Department, Fermi National Accelerator Laboratory, \\ P.O. Box 500, Batavia, IL 60510, U.S.A. \\ E-mail: brivio@thphys.uni-heidelberg.de, \\ kristian.p.moffat@durham.ac.uk, silvia.pascoli@durham.ac.uk, \\ petcov@sissa.it, jturner@fnal.gov
}

ERRATUM TO: JHEP10(2019)059

ARXIV EPRINT: 1905.12642

The related work by Vedran Brdar, Alexander J. Helmboldt, Sho Iwamoto and Kai Schmitz, who report consistent results on the viability of leptogenesis in the Neutrino Option [76] and examine possible embeddings of the Neutrino Option in a conformal theory [77, 78], was erroneously not cited in the original version of this paper.

Open Access. This article is distributed under the terms of the Creative Commons Attribution License (CC-BY 4.0), which permits any use, distribution and reproduction in any medium, provided the original author(s) and source are credited.

\section{References}

[76] V. Brdar, A.J. Helmboldt, S. Iwamoto and K. Schmitz, Type-I Seesaw as the Common Origin of Neutrino Mass, Baryon Asymmetry and the Electroweak Scale, Phys. Rev. D 100 (2019) 075029 [arXiv:1905.12634] [INSPIRE].

[77] V. Brdar, Y. Emonds, A.J. Helmboldt and M. Lindner, Conformal Realization of the Neutrino Option, Phys. Rev. D 99 (2019) 055014 [arXiv:1807.11490] [InSPIRE].

[78] V. Brdar, A.J. Helmboldt and J. Kubo, Gravitational Waves from First-Order Phase Transitions: LIGO as a Window to Unexplored Seesaw Scales, JCAP 02 (2019) 021 [arXiv: 1810.12306] [INSPIRE]. 